\title{
Asking a big question with a small satellite
}

\author{
HaloSat, NASA's first astrophysics-focused CubeSat mission - the size of a small briefcase - will survey the \\ Milky Way's halo in order to assess its complement of hot baryons, explains Principal Investigator Philip Kaaret.
}

O bservations of the cosmic microwave background show that the baryons in the 400,000-year-old Universe were at a temperature of about $3,000 \mathrm{~K}$ and constituted $4.87 \% \pm 0.19 \%$ of the total mass/energy of the early Universe ${ }^{1}$. Astronomical measurements of the presentday Universe find baryons over a wide range of temperatures but reveal only two-thirds of the baryons seen in the early Universe ${ }^{2}$. HaloSat, NASA's first astrophysics-focused CubeSat mission, will attempt to address this 'missing baryon' problem. The missing baryons are likely in a hot gas phase of millions of Kelvin in haloes surrounding galaxies, large-scale filaments between galaxies, or some combination of the two. HaloSat will observe the hot halo of our Galaxy and help to estimate the contribution of hot galactic haloes to the current baryon budget. Accurate measurement of the halo's properties may also constrain models of how the Milky Way formed, providing insight into the roles of accretion from the intergalactic medium and stellar feedback from the Galaxy in creating and powering the halo.

Line emission and absorption from highly ionized oxygen, $\mathrm{O}$ VII and $\mathrm{O}$ viII, are the primary diagnostics to study $\sim 10^{6} \mathrm{~K}$ gas. HaloSat will map line emission from $\mathrm{O}$ vII and $\mathrm{O}$ VIII in the $500-700 \mathrm{eV}$ soft $\mathrm{X}$-ray range across the whole sky using commercially available silicon drift detectors. A very modest angular resolution, on the order of $15^{\circ}$, will suffice to determine whether the halo is roughly spherical, and thus extended and massive, or flattened, and thus compact and not massive. The all-sky survey will also enable studies of large-angular-scale features in the soft X-ray sky such as the North Polar Spur, a hot interstellar bubble created by winds of massive stars and supernova explosions, and the Galactic Bulge emission, which may be powered by the Galaxy's central supermassive black hole.

The figure of merit for observing diffuse emission, survey grasp, is the product of the effective telescope collecting area multiplied by the angular area of the telescope's field of view. HaloSat uses three small detectors with a total effective area of $25 \mathrm{~mm}^{2}$ at $600 \mathrm{eV}$, but its field of view of about $100 \mathrm{deg}^{2}$ enables it to efficiently survey the entire sky in a one-year mission. Current emission-line measurements of the halo are limited in accuracy by foreground emission from

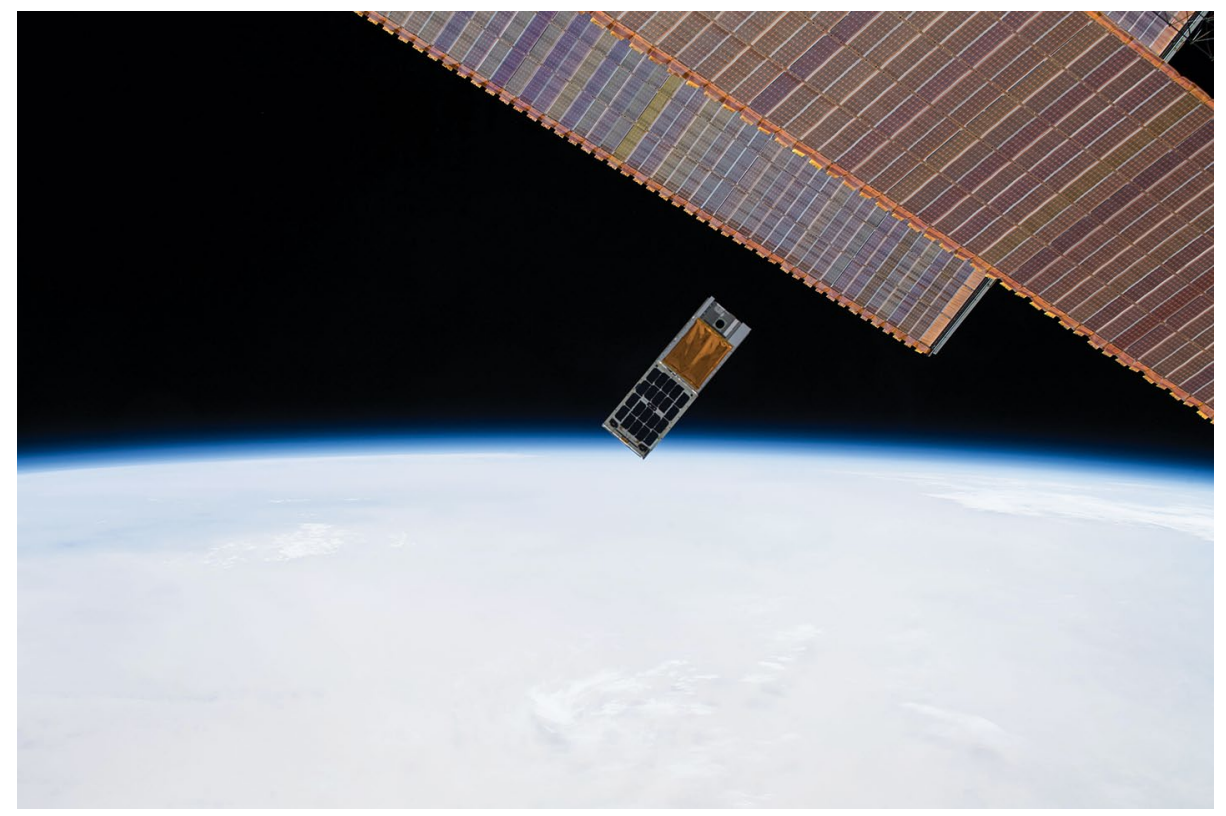

Fig. 1 | HaloSat just after deployment from the ISS. HaloSat and RainCube, a CubeSat from the Jet Propulsion Laboratory, are seen in the centre. RainCube is in front of the Earth's limb, while HaloSat has a gold thermal shield. The two CubeSats have identical dimensions while stowed and are touching when deployed, but not physically attached. They separated a few minutes after this photograph was taken and are now tens of kilometres apart. Parts of the ISS's solar array are visible in the upper right. Credit: NASA

oxygen atoms within the Solar System, which are ionized by energetic particles in the solar wind. HaloSat observes towards the anti-Sun direction during the night-time half of its 93-minute orbit around Earth to minimize this foreground.

HaloSat measures $10 \times 22 \times 36 \mathrm{~cm}$ before its solar array is unfurled, and has a mass of $12 \mathrm{~kg}$, meeting the CubeSat standard originated by Jordi Puig-Suari and Bob Twiggs in 1999. Standard physical dimensions make CubeSats interchangeable, decoupling satellite design from specific rocket launch opportunities. This has greatly increased the frequency of small-satellite launches while decreasing their cost and has driven the rapid commercialization of small-satellite technologies. HaloSat exploits a commercial CubeSat 'bus' that provides control, pointing, power, and communications systems. Engineering labour is typically the largest cost in space mission development. Re-use of components amortizes engineering costs over multiple missions, thus enabling low-cost, yet capable, small-satellite-based science missions.
HaloSat was developed on a rapid timescale with funding starting in January 2016, leading to a launch on 23 May 2018 on the Orbital ATK OA-9 International Space Station (ISS) resupply mission. HaloSat was deployed from the ISS on 13 July 2018 (Fig. 1). At the time of writing, we are commissioning the spacecraft in preparation for a one-year science observing programme. HaloSat is led by the University of Iowa in partnership with NASA's Wallops Flight Facility, NASA's Goddard Space Flight Center, Blue Canyon Technologies, Johns Hopkins University, and Université Paris-Saclay.

\section{Philip Kaaret \\ University of Iowa, Iowa City, IA, USA. \\ e-mail:philip-kaaret@uiowa.edu}

Published online: 3 September 2018 https://doi.org/10.1038/s41550-018-0571-4

1. Planck Collaboration. Astron. Astrophys. 571, Al6 (2014)

2. Shull, J. M., Smith, B. D. \& Danforth, C. W. Astrophys. J. 759,
} 23 (2012). 